Article

\title{
Inequalities on Sasakian Statistical Manifolds in Terms of Casorati Curvatures
}

\author{
Chul Woo Lee ${ }^{1}$ and Jae Won Lee ${ }^{2, *}$ \\ 1 Department of Mathematics, Kyungpook National University, Daegu 41566, Korea; mathisu@knu.ac.kr \\ 2 Department of Mathematics Education and RINS, Gyeongsang National University, Jinju 52828, Korea \\ * Correspondence: leejaew@gnu.ac.kr; Tel.: +82-55-772-2251
}

Received: 13 September 2018; Accepted: 15 November 2018; Published: 17 November 2018

\begin{abstract}
A statistical structure is considered as a generalization of a pair of a Riemannian metric and its Levi-Civita connection. With a pair of conjugate connections $\nabla$ and $\nabla^{*}$ in the Sasakian statistical structure, we provide the normalized scalar curvature which is bounded above from Casorati curvatures on C-totally real (Legendrian and slant) submanifolds of a Sasakian statistical manifold of constant $\varphi$-sectional curvature. In addition, we give examples to show that the total space is a sphere.
\end{abstract}

Keywords: Sasakian statistical manifold; conjugate connection; Casorati curvature

\section{Introduction}

A statistical model in information geometry has a Fisher metric as a Riemannian metric with an affine connection, whose connection is constructed from the average of the probability distribution. In the statistical models, a pair of a Fisher information metric and an affine connection gives the geometric structure, called the Chentsov-Amari connection [1], whose geometric structure is a generalization of a pair of a Riemannian metric and a Levi-Civita connection. By generalizing the geometric structure, a statistical structure has been studied in information geometry. Applying this idea to Sasakian manifolds, one arrived at the definition of a Sasakian statistical structure as a generalization of a Sasakian structure. In other words, it is a triple of an affine connection, a Riemannian metric, and a Sasakian structure on an odd dimensional manifold [2]. The geometry of such a manifold is closely related to affine geometry and Hessian geometry. In such manifolds, there are the fundamental equations such as Gauss formula, Weingarten formula and the equations of Gauss, Codazzi and Ricci in submanifolds of a statistical manifold [3].

On the other hand, it is well-known that the Casorati curvature as a new extrinsic invariant is defined as the normalized square of the length of the second fundamental form, introduced by Casorati $([4,5])$. Geometric meanings of Casorati curavature were found in visual perception of shape and appearance ([6-8]). Some optimal inequalities involving Casorati curvatures were proved in [9-15] for several submanifolds in real, complex and quaternionic space forms with various connections. Moreover, Lee et al. established that the normalized scalar curvature is bounded by Casorati curvatures of submanifolds in a statistical manifold of constant curvature [16]. In Kenmotsu statistical manifolds, Decu et al. investigate curvature properties and establish optimizations in terms of a new extrinsic invariant (the normalized $\delta$-Casorati curvature) and an intrinsic invariant (the scalar curvature) [17].

In our paper, we establish optimizations of the normalized scalar curvature (the intrinsic invariant) for a new extrinsic invariant (generalized normalized Casorati curvatures) on Legendrian and slant submanifolds in a Sasakian statistical space form. Moreover, we provide some examples for special Sasakian statistical sphere $S^{2 m+1}$ of statistical sectional curvature 1 . 


\section{Preliminaries}

Let $\left(\bar{M}^{m}, \bar{g}\right)$ be a $m$-dimensional Riemannian manifold with an affine connection $\bar{\nabla}$. We denote by $\Gamma(T \bar{M})$ the collection of all vector fields on $\bar{M}$.

Definition 1 ([18]). A pair $(\bar{\nabla}, \bar{g})$ is called a statistical structure on $M$ if $\bar{\nabla}$ is a torsion free connection on $M$ and the covariant derivative $\nabla g$ is symmetric.

Definition 2. A statistical manifold $(\bar{M}, \bar{g}, \bar{\nabla})$ is a Riemannian manifold, endowed with a pair of torsion-free affine connections $\bar{\nabla}$ and $\bar{\nabla}^{*}$ satisfying

$$
Z \bar{g}(X, Y)=\bar{g}\left(\bar{\nabla}_{Z} X, Y\right)+\bar{g}\left(X, \bar{\nabla}_{Z}^{*} Y\right)
$$

for any vector fields $X, Y$ and $Z$. The connections $\bar{\nabla}$ and $\bar{\nabla}^{*}$ are called dual connections.

\section{Remark 1.}

(a) $\left(\bar{\nabla}^{*}\right)^{*}=\bar{\nabla}$.

(b) If $(\bar{\nabla}, \bar{g})$ is a statistical structure, then so is $\left(\bar{\nabla}^{*}, g\right)$.

(c) Any torsion-free affine connection $\bar{\nabla}$ always has a dual connection satisfying

$$
\bar{\nabla}+\bar{\nabla}^{*}=2 \bar{\nabla}^{0}
$$

where $\bar{\nabla}^{0}$ is the Levi-Civita connection for $\bar{M}$.

Let $\bar{R}$ and $\bar{R}^{*}$ be the curvature tensor fields of $\bar{\nabla}$ and $\bar{\nabla}^{*}$, respectively.

Definition 3 ([18,19]). Let $(\bar{\nabla}, \bar{g})$ be a statistical structure on $\bar{M}$. We define

$$
S(X, Y) Z=\frac{1}{2}\left\{\bar{R}(X, Y) Z+\bar{R}^{*}(X, Y) Z\right\}
$$

for $X, Y, Z \in \Gamma(T \bar{M})$, called the statistical curvature tensor of $(\bar{\nabla}, \bar{g})$. In particular, a statistical manifold $(\bar{M}, \bar{\nabla}, \bar{g})$ is to be of constant statistical curvature $c \in \mathbb{R}$ if $S(X, Y) Z=c\{\bar{g}(Y, Z) X-\bar{g}(X, Z) Y\}$ for $X, Y, Z \in \Gamma(T \bar{M})$.

By the direct calculation, the curvature tensor fields $\bar{R}$ and $\bar{R}^{*}$ satisfy

$$
\bar{g}\left(\bar{R}^{*}(X, Y) Z, W\right)=-\bar{g}(Z, \bar{R}(X, Y) W), \quad X, Y, Z, W \in \Gamma(T \bar{M})
$$

Therefore, if $(\bar{\nabla}, \bar{g})$ is a statistical structure of constant curvature $c$, so is $\left(\bar{\nabla}^{*}, \bar{g}\right)$.

For submanifolds in statistical manifolds, we have pairs of induced connections $\nabla, \nabla^{*}$, second fundamental forms $h, h^{*}$, shape operators $A, A^{*}$, and normal connections $D, D^{*}$ satisfying equations analogous to the Gauss and the Weingarten ones for $\bar{\nabla}$ and $\bar{\nabla}^{*}$, respectively. Moreover, the induced metric $g$ is unique, and $(\nabla, g)$ and $\left(\nabla^{*}, g\right)$ are induced dual statistical structures on the submanifold. The fundamental equations for statistical submanifolds are given by Vos ([3]).

Let $(M, g)$ be an $n$-dimensional submanifold of a statistical manifold $(\bar{M}, \bar{g})$ and $g$ the induced metric on $M$. Then for any vector fields $X, Y$, the Gauss formulas are given respectively by

$$
\begin{aligned}
& \bar{\nabla}_{X} Y=\nabla_{X} Y+h(X, Y) \\
& \bar{\nabla}_{X}^{*} Y=\nabla_{X}^{*} Y+h^{*}(X, Y)
\end{aligned}
$$


The corresponding Gauss equations with respect to $\bar{\nabla}$ and $\bar{\nabla}^{*}$ are given by the following result.

Theorem 1 ([3]). Let $\bar{\nabla}$ and $\bar{\nabla}^{*}$ be dual connections on $(\bar{M}, \bar{g})$ and $\nabla$ and $\nabla^{*}$ the induced dual connections by $\bar{\nabla}$ and $\bar{\nabla}^{*}$ by a submanifold $M$ of $(\bar{M}, \bar{g})$, respectively. Let $\bar{R}, R, \overline{R^{*}}$ and $R^{*}$ be the Riemannian curvature tensors of $\bar{\nabla}, \nabla, \bar{\nabla}^{*}$ and $\nabla^{*}$, respectively. Then

$$
\begin{aligned}
\bar{g}(\bar{R}(X, Y) Z, W) & =\bar{g}(R(X, Y) Z, W) \\
& +\bar{g}\left(h(X, Z), h^{*}(Y, W)\right)-\bar{g}\left(h^{*}(X, W), h(Y, Z)\right) \\
\bar{g}\left(\bar{R}^{*}(X, Y) Z, W\right) & =\bar{g}\left(R^{*}(X, Y) Z, W\right) \\
& +\bar{g}\left(h^{*}(X, Z), h(Y, W)\right)-\bar{g}\left(h(X, W), h^{*}(Y, Z)\right)
\end{aligned}
$$

If $\left\{e_{1}, \ldots, e_{n}\right\}$ is an orthonormal basis of the tangent space $T_{p} M$ and $\left\{e_{n+1}, \ldots, e_{m}\right\}$ is an orthonormal basis of the normal space $T_{p}^{\perp} M$, then the scalar curvature $\tau$ at $p$ is defined as

$$
\tau(p)=\sum_{1 \leq i<j \leq n} g\left(S\left(e_{i}, e_{j}\right) e_{j}, e_{i}\right)
$$

and the normalized scalar curvature $\rho$ of $M$ is defined as

$$
\rho=\frac{2 \tau}{n(n-1)}
$$

We denote by $H, H^{*}$ the mean curvature vectors, that is,

$$
H(p)=\frac{1}{n} \sum_{i=1}^{n} h\left(e_{i}, e_{i}\right), \quad H^{*}(p)=\frac{1}{n} \sum_{i=1}^{n} h^{*}\left(e_{i}, e_{i}\right)
$$

and we also set

$$
h_{i j}^{\alpha}=g\left(h\left(e_{i}, e_{j}\right), e_{\alpha}\right), \quad h_{i j}^{* \alpha}=g\left(h^{*}\left(e_{i}, e_{j}\right), e_{\alpha}\right),
$$

$i, j \in\{1, \ldots, n\}, \alpha \in\{n+1, \ldots, m\}$.

Then it is well-known that the squared mean curvatures of the submanifold $M$ in $\bar{M}$ are defined by

$$
\|H\|^{2}=\frac{1}{n^{2}} \sum_{\alpha=n+1}^{m}\left(\sum_{i=1}^{n} h_{i i}^{\alpha}\right)^{2}, \quad\left\|H^{*}\right\|^{2}=\frac{1}{n^{2}} \sum_{\alpha=n+1}^{m}\left(\sum_{i=1}^{n} h_{i i}^{* \alpha}\right)^{2}
$$

and the squared norms of $h$ and $h^{*}$ over dimension $n$ is denoted by $\mathcal{C}$ and $\mathcal{C}^{*}$ are called the Casorati curvatures of the submanifold $M$, respectively. Therefore, we have

$$
\mathcal{C}=\frac{1}{n} \sum_{\alpha=n+1}^{m} \sum_{i, j=1}^{n}\left(h_{i j}^{\alpha}\right)^{2} \text { and } \mathcal{C}^{*}=\frac{1}{n} \sum_{\alpha=n+1}^{m} \sum_{i, j=1}^{n}\left(h_{i j}^{* \alpha}\right)^{2} .
$$

The normalized $\delta$-Casorati curvatures $\delta_{C}(n-1)$ and $\widehat{\delta}_{C}(n-1)$ of the submanifold $M$ are defined as

$$
\left[\delta_{C}(n-1)\right]_{p}=\frac{1}{2} \mathcal{C}_{p}+\frac{(n+1)}{2 n} \inf \left\{\mathcal{C}(L) \mid L \text { a hyperplane of } T_{p} M\right\}
$$

and

$$
\left[\widehat{\delta}_{C}(n-1)\right]_{p}=2 \mathcal{C}_{p}-\frac{(2 n-1)}{2 n} \sup \left\{\mathcal{C}(L) \mid L \text { a hyperplane of } T_{p} M\right\}
$$


Similarly, the dual normalized $\delta^{*}$-Casorati curvatures $\delta_{C}^{*}(n-1)$ and $\widehat{\delta}_{C}^{*}(n-1)$ of the submanifold $M$ are defined as

$$
\left[\delta_{C}^{*}(n-1)\right]_{p}=\frac{1}{2} \mathcal{C}_{p}^{*}+\frac{(n+1)}{2 n} \inf \left\{\mathcal{C}^{*}(L) \mid L \text { a hyperplane of } T_{p} M\right\},
$$

and

$$
\left[\widehat{\delta}_{C}^{*}(n-1)\right]_{p}=2 \mathcal{C}_{p}^{*}-\frac{(2 n-1)}{2 n} \sup \left\{\mathcal{C}^{*}(L) \mid L \text { a hyperplane of } T_{p} M\right\} .
$$

The generalized normalized $\delta$-Casorati curvatures $\delta_{C}(t ; n-1)$ and $\widehat{\delta}_{C}(t ; n-1)$ of the submanifold $M$ are defined for any positive real number $t \neq n(n-1)$ as

$$
\left[\delta_{C}(t ; n-1)\right]_{p}=t \mathcal{C}_{p}+\frac{(n-1)(n+t)\left(n^{2}-n-t\right)}{n t} \inf \left\{\mathcal{C}(L) \mid L \text { a hyperplane of } T_{p} M\right\},
$$

if $0<t<n^{2}-n$, and

$$
\left[\widehat{\delta}_{C}(t ; n-1)\right]_{p}=t \mathcal{C}_{p}-\frac{(n-1)(n+t)\left(t-n^{2}+n\right)}{n t} \sup \left\{\mathcal{C}(L) \mid L \text { a hyperplane of } T_{p} M\right\},
$$

if $t>n^{2}-n$.

Moreover, the dual generalized normalized $\delta$-Casorati curvatures $\delta_{C}^{*}(t ; n-1)$ and $\widehat{\delta}_{C}^{*}(t ; n-1)$ of the submanifold $M$ are defined for any positive real number $t \neq n(n-1)$ as

$$
\left[\delta_{C}^{*}(t ; n-1)\right]_{p}=t \mathcal{C}_{p}^{*}+\frac{(n-1)(n+t)\left(n^{2}-n-t\right)}{n t} \inf \left\{\mathcal{C}^{*}(L) \mid L \text { a hyperplane of } T_{p} M\right\},
$$

if $0<t<n^{2}-n$, and

$$
\left[\widehat{\delta}^{*} C(t ; n-1)\right]_{p}=t \mathcal{C}_{p}^{*}-\frac{(n-1)(n+t)\left(t-n^{2}+n\right)}{n t} \sup \left\{\mathcal{C}^{*}(L) \mid L \text { a hyperplane of } T_{p} M\right\},
$$

if $t>n^{2}-n$.

The following lemma plays a key role in the proof of our main theorem.

Lemma 1 ([20]). Let

$$
\Gamma=\left\{\left(x_{1}, x_{2}, \cdots, x_{n}\right) \in \mathbb{R}^{n}: x_{1}+x_{2}+\cdots+x_{n}=k\right\}
$$

be a hyperplane of $\mathbb{R}^{n}$, and $f: \mathbb{R}^{n} \longrightarrow \mathbb{R}$ a quadratic form, given by

$$
f\left(x_{1}, x_{2}, \cdots, x_{n}\right)=a \sum_{i=1}^{n-1}\left(x_{i}\right)^{2}+b\left(x_{n}\right)^{2}-2 \sum_{1 \leq i<j \leq n} x_{i} x_{j}, \quad a>0, b>0 .
$$

Then, the constrained extremum problem $\min _{x \in \Gamma} f(x)$ has a global solution as follows:

$$
x_{1}=x_{2}=\cdots=x_{n-1}=\frac{k}{a+1}, \quad x_{n}=\frac{k}{b+1}=\frac{k(n-1)}{(a+1) b}=(a-n+2) \frac{k}{a+1},
$$

provided that

$$
b=\frac{n-1}{a-n+2} .
$$


Definition 4. A triple $(\bar{g}, \varphi, \xi)$ is called an almost contact metric structure on $\bar{M}$ if the following equations hold

$$
\varphi \xi=0, \quad \bar{g}(\xi, \xi)=1, \quad \varphi^{2} X=-X+\bar{g}(X, \xi) \xi, \quad \bar{g}(\varphi X, Y)+\bar{g}(X, \varphi Y)=0, \quad X, Y \in \Gamma(T \bar{M})
$$

where $\varphi$ is a section of $T \bar{M} \otimes T \bar{M}^{*}$ and $\xi$ is the structure vector field on $\bar{M}$.

Definition 5. A quadraple $(\bar{\nabla}, \bar{g}, \varphi, \xi)$ is called a Sasakian statistical structure on $\bar{M}$ if $(\bar{\nabla}, \bar{g})$ is a statistical structure.

Theorem 2 ([2]). Let $(\bar{\nabla}, \bar{g}, \varphi, \xi)$ be a Sasakian statistical structure on $\bar{M}$. Then, so is $\left(\bar{\nabla}^{*}, \bar{g}, \varphi, \xi\right)$.

Definition 6. Let $(\bar{\nabla}, \bar{g}, \varphi, \xi)$ be a Sasakian statistical structure on $\bar{M}$, and $c \in \mathbb{R}$. The Sasakian statistical structure is said to be of constant $\varphi$-sectional curvature if

$$
\begin{aligned}
S(X, Y) Z & =\frac{c+3}{4}\{\bar{g}(Y, Z) X-\bar{g}(X, Z) Y\}+\frac{c-1}{4}\{\bar{g}(\varphi Y, Z) \varphi X \\
& -\bar{g}(\varphi X, Z) \varphi Y-2 \bar{g}(\varphi X, Y) \varphi Z-\bar{g}(Y, \bar{\xi}) \bar{g}(Z, \xi) X \\
& +\bar{g}(X, \xi) \bar{g}(Z, \xi) Y+\bar{g}(Y, \xi) \bar{g}(Z, X) \xi-\bar{g}(X, \xi) \bar{g}(Z, Y) \xi\}
\end{aligned}
$$

$X, Y, Z \in \Gamma(T \bar{M})$

A submanifold $M^{n}$ normal to $\xi$ in a Sasakian statistical manifold $\bar{M}^{2 m+1}$ is said to be a C-totally real submanifold. In this case, $\varphi\left(T_{p} M\right) \subset T_{p}^{\perp} M, p \in M$. In particular, if $n=m$, then $M$ is called a Legendrian submanifold.

For submanifolds tangent to $\xi$, there is a $\theta$-slant submanifold of a Sasakian statistical manifold as follows [21]:

A submanifold $M^{n}$ tangent to $\xi$ in a Sasakian statistical manifold is called a $\theta$-slant submanifold if for any vector $X \in T_{p} M$, linearly independent on $\xi_{p}$, the angle between $\varphi X$ and $T_{p} M$ is a constant $\theta \in\left[0, \frac{\pi}{2}\right]$, called the slant angle of $M$ in $\bar{M}$. In particular, if $\theta=0$ and $\theta=\frac{\pi}{2}, M$ is invariant and anti-invariant, respectively.

\section{Inequalities with Casorati Curvatures}

Let $M$ be an $n$-dimensional C-totally real submanifold of a $(2 m+1)$-dimensional Sasakian statistical manifold $(\bar{M}, \bar{\nabla}, \bar{g}, \varphi, \xi)$.

Let $p \in M$ and the set $\left\{e_{1}, e_{2}, \cdots, e_{n}\right\}$ and $\left\{e_{n+1}, e_{n+2}, \cdots, e_{2 m}, e_{2 m+1}=\xi\right\}$ be orthonormal bases of $T_{p} M$ and $T_{p}^{\perp} M$, respectively. Then, we have the scalar curvature as follows:

$$
\begin{aligned}
2 \tau(p) & =2 \sum_{1 \leq i<j \leq n} g\left(S\left(e_{i}, e_{j}\right) e_{j}, e_{i}\right) \\
& =\sum_{1 \leq i<j \leq n}\left\{\bar{g}\left(R\left(e_{i}, e_{j}\right) e_{j}, e_{i}\right)+\bar{g}\left(R^{*}\left(e_{i}, e_{j}\right) e_{j}, e_{i}\right)\right\} \\
& =\sum_{1 \leq i<j \leq n}\left\{\frac{c+3}{2}+\bar{g}\left(h\left(e_{i}, e_{i}\right), h^{*}\left(e_{j}, e_{j}\right)\right)+\bar{g}\left(h^{*}\left(e_{i}, e_{i}\right), h\left(e_{j}, e_{j}\right)\right)\right. \\
& \left.-2 \bar{g}\left(h^{*}\left(e_{i}, e_{j}\right), h\left(e_{i}, e_{j}\right)\right)\right\} \\
& =\frac{n(n-1)(c+3)}{4}+n^{2} \bar{g}\left(H, H^{*}\right)-\sum_{i, j=1}^{n} \bar{g}\left(h^{*}\left(e_{i}, e_{j}\right), h\left(e_{i}, e_{j}\right)\right)
\end{aligned}
$$

Since $2 H^{0}=H+H^{*}$ and the definition of Casorati curvature, $4\left\|H^{0}\right\|^{2}=\|H\|^{2}+\left\|H^{*}\right\|^{2}+$ $2 g\left(H, H^{*}\right)$, we obtain that 


$$
\begin{aligned}
2 \tau(p) & =\frac{n(n-1)(c+3)}{4}+2 n^{2}\left\|H^{0}\right\|^{2} \\
& -\frac{n^{2}}{2}\left(\|H\|^{2}+\left\|H^{*}\right\|^{2}\right)-2 n \mathcal{C}^{0}+\frac{n}{2}\left(\mathcal{C}+\mathcal{C}^{*}\right)
\end{aligned}
$$

where $\mathcal{C}^{0}=\frac{1}{2}\left(\mathcal{C}+\mathcal{C}^{*}\right)$

Define a quadratic polynomial in the components of the second fundamental form $h^{0}$ by

$$
\begin{aligned}
\mathcal{P}= & t \mathcal{C}^{0}+\frac{(n-1)(n+t)\left(n^{2}-n-t\right)}{n t} \mathcal{C}^{0}(L)+\frac{1}{2} n\left(\mathcal{C}+\mathcal{C}^{*}\right) \\
& -\frac{n^{2}}{2}\left(\|H\|^{2}+\left\|H^{*}\right\|^{2}\right)-2 \tau(p)+\frac{n(n-1)(c+3)}{4}
\end{aligned}
$$

where $L$ is a hyperplane of $T_{p} M$. Without loss of generality, we can assume that $L$ is spanned by $e_{1}, \cdots, e_{n-1}$. Then we derive

$$
\begin{aligned}
\frac{1}{2} \mathcal{P} & =\sum_{\alpha=n+1}^{m} \sum_{i=1}^{n-1}\left[\frac{n^{2}+n(t-1)-2 t}{r}\left(h_{i i}^{0 \alpha}\right)^{2}+\frac{2(n+t)}{n}\left(h_{i n}^{0 \alpha}\right)^{2}\right] \\
& +\sum_{\alpha=n+1}^{m}\left[\frac{2(n+t)(n-1)}{t} \sum_{1=i<j}^{n-1}\left(h_{i j}^{0 \alpha}\right)^{2}-2 \sum_{1=i<j}^{n} h_{i i}^{0 \alpha} h_{j j}^{0 \alpha}+\frac{t}{n}\left(h_{n n}^{0 \alpha}\right)^{2}\right] \\
& \geq \sum_{\alpha=n+1}^{m}\left[\sum_{i=1}^{n-1} \frac{n^{2}+n(t-1)-2 t}{t}\left(h_{i i}^{0 \alpha}\right)^{2}-2 \sum_{1=i<j}^{n} h_{i i}^{0 \alpha} h_{j j}^{0 \alpha}+\frac{t}{n}\left(h_{n n}^{0 \alpha}\right)^{2}\right] .
\end{aligned}
$$

For $\alpha=n+1, \cdots, m$, let us consider the quadratic form $f_{\alpha}: \mathbb{R}^{n} \longrightarrow \mathbb{R}$ defined by

$$
\begin{aligned}
f_{\alpha}\left(h_{11}^{0 \alpha}, \ldots, h_{n n}^{0 \alpha}\right) & =\frac{n^{2}+n(t-1)-2 t}{t} \sum_{i=1}^{n-1}\left(h_{i i}^{0 \alpha}\right)^{2} \\
& -2 \sum_{1=i<j}^{n} h_{i i}^{0 \alpha} h_{j j}^{0 \alpha}+\frac{t}{n}\left(h_{n n}^{0 \alpha}\right)^{2},
\end{aligned}
$$

and the constrained extremum problem

$$
\begin{gathered}
\min f_{\alpha} \\
\text { subject to } F^{\alpha}: h_{11}^{0 \alpha}+\cdots+h_{n n}^{0 \alpha}=c^{\alpha},
\end{gathered}
$$

where $c^{\alpha}$ is a real constant. Comparing (10) with the quadratic function in Lemma 1, we see that

$$
a=\frac{n^{2}+n(t-1)-2 t}{t}, \quad b=\frac{t}{n} .
$$

Therefore, we have the critical point $\left(h_{11}^{0 \alpha}, \cdots, h_{n n}^{0 \alpha}\right)$, given by

$$
h_{11}^{0 \alpha}=h_{22}^{0 \alpha}=\cdots=h_{n-1 n-1}^{0 \alpha}=\frac{t c^{\alpha}}{(n+t)(n-1)}, \quad h_{n n}^{0 \alpha}=\frac{n c^{\alpha}}{n+t},
$$

is a global minimum point by Lemma 1 . Moreover, $f_{\alpha}\left(h_{11}^{0 \alpha}, \cdots, h_{n n}^{0 \alpha}\right)=0$. Therefore, we have

$$
\mathcal{P} \geq 0,
$$

which implies

$$
\begin{aligned}
2 \tau(p) \leq & t \mathcal{C}^{0}+\frac{(n-1)(n+t)\left(n^{2}-n-t\right)}{n t} \mathcal{C}^{0}(L)+\frac{1}{2} n\left(\mathcal{C}+\mathcal{C}^{*}\right) \\
& -\frac{n^{2}}{2}\left(\|H\|^{2}+\left\|H^{*}\right\|^{2}\right)+\frac{n(n-1)(c+3)}{4}
\end{aligned}
$$


Therefore, we derive

$$
\begin{aligned}
\rho & \leq \frac{1}{n(n-1)}\left\{t \mathcal{C}^{0}+\frac{(n-1)(n+t)\left(n^{2}-n-t\right)}{n t} \mathcal{C}^{0}(L)\right\} \\
& +\frac{1}{2(n-1)}\left(\mathcal{C}+\mathcal{C}^{*}\right)-\frac{n}{2(n-1)}\left(\|H\|^{2}+\left\|H^{*}\right\|^{2}\right)+\frac{c+3}{4} .
\end{aligned}
$$

Therefore, we have the following theorem:

Theorem 3. Let $M$ be an n-dimensional $C$-totally real submanifold of a $(2 m+1)$-dimensional Sasakian statistical manifold $(\bar{M}, \bar{\nabla}, \bar{g}, \varphi, \xi)$. When $0<t<n^{2}-n$, the generalized normalized $\delta$-Casorati curvature $\delta_{C}^{0}(t, n-1)$ on $M$ satisfies

$$
\begin{aligned}
\rho & \leq \frac{1}{n(n-1)} \delta_{C}^{0}(t, n-1)+\frac{1}{2(n-1)}\left(\mathcal{C}+\mathcal{C}^{*}\right) \\
& -\frac{n}{2(n-1)}\left(\|H\|^{2}+\left\|H^{*}\right\|^{2}\right)+\frac{c+3}{4}
\end{aligned}
$$

where $2 \delta_{C}^{0}(t, n-1)=\delta_{C}(t, n-1)+\delta_{C}^{*}(t, n-1)$. The equality case holds identically at any point $p \in M$ if and only if $h=-h^{*}$.

For a unit hypersphere $S^{2 n+1}$ in $\mathbb{R}^{2 n+2}$, the unit normal vector field $N$ of $S^{2 n+1}$ provides the structure vector field $\xi=-J N$ with the standard almost complex structure $J$ on $\mathbb{R}^{2 n+2}=\mathbb{C}^{n+1}$. In addition, $\varphi=\pi \circ \mathrm{J}$ is the natural projection of the tangent space of $\mathbb{R}^{2 n+2}$ onto the tangent space of $S^{2 n+1}$. Then we obtain the standard Sasakian structure $(g, \varphi, \xi)$ on $S^{2 n+1}$. From [2], we can construct a Sasakian statistical structures on $S^{2 n+1}$ of constant statistical sectional curvature 1 . Therefore, we have the following optimal inequality:

Example 1. Let $M$ be an $n$-dimensional C-totally real submanifold of $S^{2 m+1}$. Then, the generalized normalized $\delta$-Casorati curvature $\delta_{C}^{0}(t, n-1)$ on $M^{n}$ satisfies

$$
\begin{aligned}
\rho & \leq \frac{1}{n(n-1)} \delta_{C}^{0}(t, n-1)+\frac{1}{2(n-1)}\left(\mathcal{C}+\mathcal{C}^{*}\right) \\
& -\frac{n}{2(n-1)}\left(\|H\|^{2}+\left\|H^{*}\right\|^{2}\right)+1
\end{aligned}
$$

When $t=\frac{n(n-1)}{2}$ in Theorem 3, we have an optimization for a normalized $\delta$-Casoratic curvature as follows:

Corollary 1. Let $M$ be an n-dimensional C-totally real submanifold of a $(2 m+1)$-dimensional Sasakian statistical manifold $(\bar{M}, \bar{\nabla}, \bar{g}, \varphi, \xi)$. Then, the normalized $\delta$-Casorati curvature $\delta_{C}^{0}(n-1)$ on M satisfies

$$
\rho \leq \delta_{C}^{0}(n-1)+\frac{1}{2(n-1)}\left(\mathcal{C}+\mathcal{C}^{*}\right)-\frac{n}{2(n-1)}\left(\|H\|^{2}+\left\|H^{*}\right\|^{2}\right)+\frac{c+3}{4} .
$$

Proof. Taking $t=\frac{n(n-1)}{2}$ in $\delta_{C}^{0}(t, n-1)$, we have the following relation:

$$
\left[\delta_{C}^{0}\left(\frac{n(n-1)}{2} ; n-1\right)\right]_{p}=n(n-1)\left[\delta_{C}^{0}(n-1)\right]_{p}
$$

in any point $p \in M$. Therefore, we have an optimal inequality for the normalized $\delta$-Casorati curvature $\delta_{C}^{0}(n-1)$. 
Theorem 4. Let $M$ be an n-dimensional $\theta$-slant submanifold of a $(2 m+1)$-dimensional Sasakian statistical manifold $(\bar{M}, \bar{\nabla}, \bar{g}, \varphi, \xi)$. When $0<t<n^{2}-n$, the generalized normalized $\delta$-Casorati curvature $\delta_{C}^{0}(t, n-1)$ on $M$ satisfies

$$
\begin{aligned}
\rho & \leq \frac{1}{n(n-1)} \delta_{C}^{0}(t, n-1)+\frac{1}{2(n-1)}\left(\mathcal{C}+\mathcal{C}^{*}\right)-\frac{n}{2(n-1)}\left(\|H\|^{2}+\left\|H^{*}\right\|^{2}\right) \\
& +\frac{n(n-1)(c+3)}{4}+\frac{3(n-1)(c-1) \cos ^{2} \theta}{4}-\frac{(n-1)(c-1)}{2} .
\end{aligned}
$$

Proof. Let $p \in M$ and the set $\left\{e_{1}, e_{2}, \cdots, e_{n-1}, e_{n}=\xi\right\}$ and $\left\{e_{n+1}, e_{n+2}, \cdots, e_{2 m}, e_{2 m+1}\right\}$ be orthonormal bases of $T_{p} M$ and $T_{p}^{\perp} M$, respectively. Then, we have the scalar curvature as follows:

$$
\begin{aligned}
2 \tau(p) & =2 \sum_{1 \leq i<j \leq n} g\left(S\left(e_{i}, e_{j}\right) e_{j}, e_{i}\right) \\
& =\sum_{1 \leq i<j \leq n}\left\{\bar{g}\left(R\left(e_{i}, e_{j}\right) e_{j}, e_{i}\right)+\bar{g}\left(R^{*}\left(e_{i}, e_{j}\right) e_{j}, e_{i}\right)\right\} \\
& =\frac{n(n-1)(c+3)}{4}+\frac{3(n-1)(c-1) \cos ^{2} \theta}{4}-\frac{(n-1)(c-1)}{2} \\
& +n^{2} \bar{g}\left(H, H^{*}\right)-\sum_{i, j=1}^{n} \bar{g}\left(h^{*}\left(e_{i}, e_{j}\right), h\left(e_{i}, e_{j}\right)\right)
\end{aligned}
$$

By using a similar argument as in the proof of Theorem 3, we get

$$
\begin{aligned}
2 \tau(p) \leq & t \mathcal{C}^{0}+\frac{(n-1)(n+t)\left(n^{2}-n-t\right)}{n t} \mathcal{C}^{0}(L) \\
& +\frac{1}{2} n\left(\mathcal{C}+\mathcal{C}^{*}\right)-\frac{n^{2}}{2}\left(\|H\|^{2}+\left\|H^{*}\right\|^{2}\right) \\
& +\frac{n(n-1)(c+3)}{4}+\frac{3(n-1)(c-1) \cos ^{2} \theta}{4}-\frac{(n-1)(c-1)}{2}
\end{aligned}
$$

Therefore, we have an ineqaulity as follows:

$$
\begin{aligned}
\rho & \leq \frac{1}{n(n-1)} \delta_{C}^{0}(t, n-1)+\frac{1}{2(n-1)}\left(\mathcal{C}+\mathcal{C}^{*}\right)-\frac{n}{2(n-1)}\left(\|H\|^{2}+\left\|H^{*}\right\|^{2}\right) \\
& +\frac{n(n-1)(c+3)}{4}+\frac{3(n-1)(c-1) \cos ^{2} \theta}{4}-\frac{(n-1)(c-1)}{2} .
\end{aligned}
$$

If $M$ is an invariant submanifold, then $\theta=0$. Then we obtain

Corollary 2. Let $M^{n}$ be an n-dimensional invariant submanifold of a $(2 m+1)$-dimensional Sasakian statistical manifold $(\bar{M}, \bar{\nabla}, \bar{g}, \varphi, \xi)$. When $0<t<n^{2}-n$, we derive

$$
\begin{aligned}
\rho & \leq \frac{1}{n(n-1)} \delta_{C}^{0}(t, n-1)+\frac{1}{2(n-1)}\left(\mathcal{C}+\mathcal{C}^{*}\right) \\
& -\frac{n}{2(n-1)}\left(\|H\|^{2}+\left\|H^{*}\right\|^{2}\right)+\frac{n(n-1)(c+3)}{4}+\frac{(n-1)(c-1)}{4} .
\end{aligned}
$$

If $M$ is an anti-invariant submanifold, then $\theta=\frac{\pi}{2}$. Then we obtain 
Corollary 3. Let $M^{n}$ be an $n$-dimensional anti-invariant submanifold of a $(2 m+1)$-dimensional Sasakian statistical manifold $(\bar{M}, \bar{\nabla}, \bar{g}, \varphi, \xi)$. When $0<t<n^{2}-n$, we derive

$$
\begin{aligned}
\rho & \leq \frac{1}{n(n-1)} \delta_{C}^{0}(t, n-1)+\frac{1}{2(n-1)}\left(\mathcal{C}+\mathcal{C}^{*}\right) \\
& -\frac{n}{2(n-1)}\left(\|H\|^{2}+\left\|H^{*}\right\|^{2}\right)+\frac{n(n-1)(c+3)}{4}-\frac{(n-1)(c-1)}{2} .
\end{aligned}
$$

Example 2. Let $M$ be an $n$-dimensional $\theta$-slant submanifold of $S^{2 m+1}$. Then, the generalized normalized $\delta$-Casorati curvature $\delta_{C}^{0}(t, n-1)$ on $M^{n}$ satisfies

$$
\begin{aligned}
\rho & \leq \frac{1}{n(n-1)} \delta_{C}^{0}(t, n-1)+\frac{1}{2(n-1)}\left(\mathcal{C}+\mathcal{C}^{*}\right) \\
& -\frac{n}{2(n-1)}\left(\|H\|^{2}+\left\|H^{*}\right\|^{2}\right)+n(n-1)
\end{aligned}
$$

\section{Remark 2.}

(1) Taking $t=\frac{n(n-1)}{2}$ as Corollary 1, we have optimal inequalities for $\theta$-slant submanifold of a Sasakian statistical manifold.

(2) In any optimization throughout our paper, the equality cases hold if and only if a submanifold is totally geodesic from $h=-h^{*}$.

(3) In the case for $t>n^{2}-n$, the methods of finding the above inequalities are analogous.

Author Contributions: C.W.L. presented the idea to establish optimizations on C-totally real (Legendrian, slant) submanifolds. J.L. checked and polished the draft.

Acknowledgments: Chul Woo Lee was supported by Basic Science Research Program through the National Research Foundation of Korea (NRF) funded by the Ministry of Education (2018R1D1A1B07040576) and Jae Won Lee was supported by the Basic Science Research Program through the National Research Foundation of Korea (NRF) funded by the Ministry of Education (2017R1D1A1B03033978).

Conflicts of Interest: The authors declare no conflict of interest.

\section{References}

1. Amari, S. Differential-Geometrical Methods in Statistics, Lecture Notes in Statistics; Springer: New York, NY, USA, 1985.

2. Furuhata, H.; Hasegawa, I.; Okuyama, Y.; Sato, K.; Shahid, M. Sasakian statistical manifolds. J. Geom. Phys. 2017, 117, 179-186. [CrossRef]

3. Vos, P.W. Fundamental equations for statistical submanifolds with applications to the Bartlett correction. Ann. Inst. Stat. Math. 1989, 41, 429-450. [CrossRef]

4. Casorati, F. Mesure de la courbure des surfaces suivant l'idée commune. Ses rapports avec les mesures de courbure gaussienne et moyenne. Acta Math. 1890, 14, 95-110. [CrossRef]

5. Kowalczyk, D. Casorati curvatures. Bull. Transilv. Univ. Brasov Ser. III 2008, 1, 209-213.

6. Koenderink, J.J. Surface shape. In The Science and the Looks in "Handbook of Experimental Phenomenology: Visual Perception of Shape, Space and Appearance"; Albertazzi, L., Ed.; John Wiley and Sons Ltd: Chichester, UK, 2013; pp. 165-180.

7. Ons, B.; Verstraelen, L. Some geometrical comments on vision and neurobiology: Seeing Gauss and Gabor walking by, when looking through the window of the Parma at Leuven in the company of Casorati. Kragujevac J. Math. 2011, 35, 317-325.

8. Verstraelen, L. Geometry of submanifolds I. The first Casorati curvature indicatrices. Kragujevac J. Math. 2013, 37, 5-23.

9. Decu, S.; Haesen, S.; Verstraelen, L. Optimal inequalities involving Casorati curvatures. Bull. Transilv. Univ. Braşov Ser. B (N.S.) 2007, 14, 85-93. 
10. Decu, S.; Haesen, S.; Verstraelen, L. Optimal inequalities characterising quasi-umbilical submanifolds. J. Inequal. Pure Appl. Math. 2008, 9, 1-7.

11. Lee, C.W.; Yoon, D.W.; Lee, J.W. Optimal inequalities for the Casorati curvatures of submanifolds of real space forms endowed with semi-symmetric metric connections. J. Inequal. Appl. 2014, 2014. [CrossRef]

12. Lee, J.W.; Vîlcu, G.-E. Inequalities for generalized normalized $\delta$-Casorati curvatures of slant submanifolds in quaternionic space forms. Taiwanese J. Math. 2015, 19, 691-702. [CrossRef]

13. Lee, C.W.; Lee, J.W.; Vîlcu, G.-E.; Yoon, D.W. Optimal inequalities for the Casorati curvatures of submanifolds of generalized space forms endowed with semi-symmetric metric connections. Bull. Korean Math. Soc. 2015, 52, 1631-1647. [CrossRef]

14. Lee, J.W.; Lee, C.W.; Yoon, D.W. Inequalities for generalized $\delta$-Casorati curvatures of submanifolds in real space forms endowed with a semi-symmetric metric connection. Rev. Union Mat. Argent. 2016, 57, $53-62$.

15. Slesar, V.; Şahin, B.; Vîlcu, G.-E. Inequalities for the Casorati curvatures of slant submanifolds in quaternionic space forms. J. Inequal. Appl. 2014, 2014. [CrossRef]

16. Lee, C.W.; Yoon, D.W.; Lee, J.W. A pinching theorem for statistical manifolds with Casorati curvatures. J. Nonlinear Sci. Appl. 2017, 10, 4908-4914. [CrossRef]

17. Decu, S.; Haesen, S.; Verstraelan, L.; Vilcu, G.-E. Curvature invariants of statistical submanifolds in Kenmotsu statistical manifolds of constant $\phi$-sectional curvature. Entropy 2018, 20, 529. [CrossRef]

18. Opozda, B. A sectional curvature for statistical structures. Linear Algebra Appl. 2016, 497, 134-161. [CrossRef]

19. Opozda, B. Bochner's technique for statistical structures. Ann. Glob. Anal. Geom. 2015, 48, 357-395. [CrossRef]

20. Tripathi, M.M. Inequalities for algebraic Casorati curvatures and their applications. Note Mater. 2017, 37, 161-186.

21. Lotta, A. Slant submanifolds in contact geometry. Bull. Math. Soc. Roum. 1996, 39, 183-198.

(C) 2018 by the authors. Licensee MDPI, Basel, Switzerland. This article is an open access article distributed under the terms and conditions of the Creative Commons Attribution (CC BY) license (http:/ / creativecommons.org/licenses/by/4.0/). 